\title{
Multiple homoclinic solutions for a class of nonhomogeneous Hamiltonian systems
}

\author{
Chunhua Deng $^{1 *}$ and Dong-Lun Wu $\mathrm{u}^{2,3}$
}

\section{"Correspondence:}

11050085@hyit.edu.cn

'Faculty of Mathematics and

Physics, Huaiyin Institute of

Technology, Huai'an, China

Full list of author information is

available at the end of the article

\begin{abstract}
By introducing a new superquadratic condition, we obtain the existence of two nontrivial homoclinic solutions for a class of perturbed second order Hamiltonian systems which are obtained by the mountain pass theorem and Ekeland's variational principle.
\end{abstract}

Keywords: Multiple Homoclinic solutions; Perturbed second order Hamiltonian systems; Superquadratic conditions; The (C) condition; Variational methods

\section{Introduction and main results}

In this paper, we consider the existence of two nontrivial homoclinic solutions for the following second order Hamiltonian systems:

$$
\ddot{u}(t)-L(t) u(t)+\nabla W(t, u(t))=f(t)
$$

for all $t \in R$, where $W \in C^{1}\left(R \times R^{N}, R\right), L: R \rightarrow R^{N^{2}}$ is a matrix-valued function and $f \in C\left(R, R^{N}\right)$. A solution $u(t)$ of problem (1) is homoclinic (to 0 ) if $u(t) \rightarrow 0$ as $t \rightarrow \pm \infty$. Moreover, if $u(t) \neq 0, u(t)$ is called a nontrivial homoclinic solution. Here and subsequently, $\nabla W(t, x)$ denotes the gradient with respect to the $x$ variable.

The homoclinic solutions have been proved to be important in studying the behavior of dynamic systems. There have been many papers concerning this topic by using the variational methods since the remarkable results by Ambrosetti and Rabinowitz [1]. Because of the lack of compactness, this problem is more difficult than studying the existence of periodic solutions. In order to get the compactness of embedding theorem back, many conditions have been proposed (see [1-41]). Two kinds of important conditions are periodic and coercive conditions. The periodic condition was introduced by Rabinowitz [19] in 1990 to discuss the existence of homoclinic solutions for problem (1) as the limit of a sequence of subharmonics which are obtained by the mountain pass theorem. The following coercive condition is a classical condition introduced by Rabinowitz and his co-author [20].

$\left(L^{\prime}\right) L \in C\left(R, R^{N^{2}}\right)$ is a symmetric and positively definite matrix for all $t \in R$ and

$$
\inf _{|x|=1}(L(t) x, x) \rightarrow+\infty \quad \text { as }|t| \rightarrow \infty
$$

(c) The Author(s) 2018. This article is distributed under the terms of the Creative Commons Attribution 4.0 International License (http://creativecommons.org/licenses/by/4.0/), which permits unrestricted use, distribution, and reproduction in any medium, provided you give appropriate credit to the original author(s) and the source, provide a link to the Creative Commons license, and indicate if changes were made. 
Condition $\left(L^{\prime}\right)$ has been studied by many other mathematicians to deal with the nonperiodic systems. After then, there have been some other coercive conditions introduced by other mathematicians.

By using the variational methods to study problem (1), the growth conditions of $W(t, x)$ are needed. These conditions are mainly classified into three cases: the superquadratic case, the subquadratic case, and the asymptotically quadratic case. In this paper, we mainly consider the superquadratic case. The following growth condition is a classical superquadratic condition known as the $(A R)$ condition.

$(A R)$ there exists a constant $\theta>2$ such that

$$
0<\theta W(t, x) \leq(\nabla W(t, x), x)
$$

for every $t \in R$ and $x \in R^{N} \backslash\{0\}$.

However, the $(A R)$ condition is so strong that many functions cannot be involved. In order to study problem (1) with different potentials, many other superquadratic conditions are proposed. In 2009, Ding and Lee [8] introduced the following generalized superquadratic condition.

(GS) There exist $\epsilon \in(0,1)$ and $r_{1}, d_{0}>0$ such that

$$
\widetilde{W}(t, x) \geq d_{0} \frac{(\nabla W(t, x), x)}{|x|^{2-\epsilon}} \quad \text { for all } t \in R \text { and }|x| \geq r_{1},
$$

where

$$
\widetilde{W}(t, x)=(\nabla W(t, x), x)-2 W(t, x) .
$$

Some examples are given to show the difference between $(G S)$ and $(A R)$ conditions. The following superquadratic condition is used by Lv and Tang [14] to obtain infinitely many homoclinic solutions for problem (1) when $W(t, x)$ is even in $x$.

$(M C)$ There exists $\varsigma \geq 1$ such that

$$
\varsigma \widetilde{W}(t, x) \geq \widetilde{W}(t, \varsigma x)
$$

for all $(t, x) \in R \times R^{N}$. Recently, Wu et al. [33] introduced the following condition:

(SQ) $\frac{\widetilde{W}(t, x)}{W(t, x)}|x|^{2} \rightarrow+\infty$ as $|x| \rightarrow \infty$ uniformly in $t \in R$, where

$$
\widetilde{W}(t, x)=(\nabla W(t, x), x)-2 W(t, x) .
$$

With $(S Q)$, the authors obtained the existence of homoclinic solutions for a class of periodic Hamiltonian systems.

In 2018, Wu et al. [32] showed the existence of homoclinic solutions for problem (1) without periodic or even conditions. In 2015, Xu et al. [36] showed the existence of two solutions for problem (1) with a nonzero perturbation. In the same year, Zhang and Yuan [40] obtained two homoclinic solutions for a class of perturbed Hamiltonian systems under the $(A R)$ condition. In this paper, we introduce a new superquadratic condition to study problem (1) with small forcing terms. The following theorem is our main result. 
Theorem 1.1 Suppose that $W$ and L satisfy the following conditions:

$(L)$ For the smallest eigenvalue of $L(t)$, i.e., $l(t) \equiv \inf _{|x|=1} L(t) x \cdot x$, there exists a constant $v<1$ such that $l(t)|t|^{\nu-2} \rightarrow+\infty$ as $|t| \rightarrow \infty$.

$(W 1) \quad W(t, 0)=0$ for all $t \in R$ and $\nabla W(t, x)=o(|x|)$ as $|x| \rightarrow 0$ uniformly for $t \in R$;

$(W 2)(\nabla W(t, x), x) \geq 2 W(t, x) \geq 0$ for all $(t, x) \in R \times R^{N}$;

(W3) $W(t, x) /|x|^{2} \rightarrow+\infty$ as $|x| \rightarrow \infty$ uniformly in $t$;

(W4) There exist $\tau>2$ and $d_{1}>0$ such that

$$
|\nabla W(t, x)| \leq d_{1}\left(1+|x|^{\tau-1}\right) \quad \text { for all } t \in R
$$

(W5) There exist constants $\mu \geq 1, \lambda_{0} \in(0,1), d_{2}>0$, and $r_{\infty}>0$ such that

$$
\left(\frac{1-\lambda^{2}}{2}-\lambda\right)(\nabla W(t, x), x)+W(t, \lambda x)-W(t, x) \geq-d_{2} \lambda^{\mu}|x|^{\mu}
$$

for all $\lambda \in\left[0, \lambda_{0}\right],|x| \geq r_{\infty}$, and $t \in R$.

Then there exists $\delta>0$ such that, for any $f \not \equiv 0$ satisfying

$$
\max _{t \in R}|f(t)| \leq \delta
$$

system (1) possesses at least two nontrivial homoclinic solutions.

Remark 1 In Theorem 1.1, the perturbation $f$ is not required to be integrable.

Remark 2 Consider the following example:

$$
G(x)=|x|^{s}+(s-2)|x|^{s-\epsilon} \sin ^{2}\left(|x|^{\epsilon} / \epsilon\right)
$$

where $s>2$ and $\epsilon \in(0, s-2)$. It is easy to check that (3) satisfies the conditions of Theorem 1.1 and but not the $(A R)$ condition. As we know, Theorem 1.1 is the first result to obtain the existence of two homoclinic solutions for problem (1) without the (AR) condition.

\section{Proof of Theorem 1.1}

Let $A$ be a self-adjoint extension of the operator $-\left(d^{2} / d t^{2}\right)+L(t)$ with the domain $\mathcal{D}(A) \subset$ $L^{2}\left(R, R^{N}\right)$. Let $E=\mathcal{D}\left(|A|^{1 / 2}\right)$ be the domain of $|A|^{1 / 2}$ and define on $E$ the inner product and the norm as $(u, w)_{0}=\left(|A|^{1 / 2} u,|A|^{1 / 2} w\right)_{2}+(u, w)_{2}$ and $\|u\|_{0}=(u, u)^{1 / 2}$, respectively, where $(\cdot, \cdot)_{2}$ denotes the inner product of $L^{2}$. Then $E$ is a Hilbert space.

It is known that the spectrum $\sigma(A)$ consists of eigenvalues numbered in $\lambda_{1} \leq \lambda_{2} \leq \cdots \rightarrow$ $\infty$, and a corresponding system of eigenfunctions $\left(e_{n}\right)\left(A e_{n}=\lambda_{n} e_{n}\right)$ forms an orthogonal basis in $L^{2}$. Let $n^{-}=\#\left\{i \mid \lambda_{i}<0\right\}, n^{0}=\#\left\{i \mid \lambda_{i}=0\right\}$, and $\bar{n}=n^{-}+n^{0}$. Set $E^{-}=\operatorname{span}\left\{e_{1}, \ldots, e_{n^{-}}\right\}$, $E^{0}=\operatorname{span}\left\{e_{n^{-}+1}, \ldots, e_{\bar{n}}\right\}$, and $E^{+}=\overline{\operatorname{span}\left\{e_{\bar{n}+1}, \ldots\right\}}$. Then $E=E^{-} \oplus E^{0} \oplus E^{+}$. The inner product and the norm on $E$ are introduced as

$$
(u, w)=\left(|A|^{1 / 2} u,|A|^{1 / 2} w\right)_{2}+\left(u^{0}, w^{0}\right)_{2}, \quad\|u\|=(u, u)^{1 / 2},
$$


where $u=u^{-}+u^{0}+u^{+}$and $w=w^{-}+w^{0}+w^{+} \in E=E^{-} \oplus E^{0} \oplus E^{+}$. Furthermore, let $I: E \rightarrow R$ be the functional defined by

$$
\begin{aligned}
I(u) & =\int_{R}\left(\frac{1}{2}|\dot{u}(t)|^{2}+\frac{1}{2}(L(t) u(t), u(t))-W(t, u(t))+(f(t), u(t))\right) d t \\
& =\frac{1}{2}\|u\|^{2}-\int_{R} W(t, u(t)) d t+\int_{R}(f(t), u(t)) d t .
\end{aligned}
$$

It is known that the critical points of $I$ in $E$ are the homoclinic solutions of (1). One can easily check that $I \in C^{1}(E, R)$ and

$$
\begin{aligned}
\left\langle I^{\prime}(u), v\right\rangle= & \int_{R}((\dot{u}(t), \dot{v}(t))+(L(t) u(t), v(t))-(\nabla W(t, u(t)), v(t))) d t \\
& +\int_{R}(f(t), v(t)) d t .
\end{aligned}
$$

By Lemma 2.2 in [6] we can conclude that $E$ is compactly embedded in $L^{p}$ for any $p \in$ $[1,+\infty]$, which implies that there exists a constant $C_{p}>0$ such that

$$
\|u\|_{L^{p}} \leq C_{p}\|u\| \quad \text { for all } u \in E
$$

Lemma 2.1 Suppose that the conditions of Theorem 1.1 hold, then there exist constants $\alpha$, $\varrho>0$ such that $\left.I\right|_{S} \geq \alpha$, where $S=\{u \in E \mid\|u\|=\varrho\}$.

Proof By (W1), for any $\varepsilon>0$, there exists $\sigma>0$ such that

$$
|\nabla W(t, x)| \leq \varepsilon|x|, \quad|x| \leq \sigma, \quad \forall t \in R
$$

Then it follows from (W1) and (W2) that

$$
\begin{aligned}
W(t, x) & =|W(t, x)-W(t, 0)| \\
& =\left|\int_{0}^{1}(\nabla W(t, s x), x) d s\right| \\
& \leq \int_{0}^{1}|\nabla W(t, s x)||x| d s \\
& \leq \int_{0}^{1} \varepsilon|s x||x| d s \\
& \leq \varepsilon|x|^{2}
\end{aligned}
$$

for all $t \in R$ and $|x| \leq \sigma$. Let $\varepsilon_{0}=\frac{1}{4 C_{2}}$, then there exists $\sigma_{0}>0$ such that (7) holds for all $t \in R$ and $|x| \leq \sigma_{0}$. Set

$$
\varrho=\frac{\sigma_{0}}{C_{\infty}}, \quad \alpha=\frac{1}{4} \varrho^{2}>0,
$$


which implies $0<\|u\|_{L^{\infty}} \leq \sigma_{0}$ for all $u \in S$. Then it follows from the definition of $I$, (7) and (2) that

$$
\begin{aligned}
I(u) & =\frac{1}{2}\|u\|^{2}-\int_{R} W(t, u(t)) d t+\int_{R}(f(t), u(t)) d t \\
& \geq \frac{1}{2}\|u\|^{2}-\frac{1}{4 C_{2}} \int_{R}|u(t)|^{2} d t-\delta \int_{R}|u(t)| d t \\
& \geq \frac{1}{4}\|u\|^{2}-\delta C_{1}\|u\| .
\end{aligned}
$$

By the definitions of $\varrho$ and $\alpha$, there exists $\delta_{0}>0$ such that $\left.I\right|_{S} \geq \alpha$ for any $f$ satisfying (2).

Lemma 2.2 Suppose that the conditions of Theorem 1.1 hold, then there is $e \in E$ such that $\|e\|>\varrho$ and $I(e) \leq 0$, where $\varrho$ is defined in Lemma 2.1 .

Proof It follows from (W3) that there exist $T>0, \xi>0$, and $\varepsilon_{1}>0$ such that

$$
W(t, x) \geq\left(\frac{\pi^{2}}{2 T^{2}}+\varepsilon_{1}\right)|x|^{2}
$$

for all $t \in[-T, T]$ and $|x|>\xi$. Set $\zeta=\max \{|W(t, x)||t \in[-T, T]| x \mid, \leq \xi\}$, hence we have

$$
W(t, x) \geq\left(\frac{\pi^{2}}{2 T^{2}}+\varepsilon_{1}\right)\left(|x|^{2}-\xi^{2}\right)-\zeta
$$

Set

$$
Q_{1}(t)= \begin{cases}\sin (\omega t) e, & t \in[-T, T], \\ 0, & t \in R \backslash[-T, T],\end{cases}
$$

where $\omega=\frac{\pi}{T}, e=(1,0, \ldots, 0)$. It can be easily checked that $\left(\frac{\pi^{2}}{2 T^{2}}+\varepsilon_{1}\right) m>M$, where

$$
M=\frac{1}{2} \int_{-T}^{T}\left|\dot{Q}_{1}(t)\right|^{2} d t, \quad m=\int_{-T}^{T}\left|Q_{1}(t)\right|^{2} d t .
$$

By (4), for every $r \in R \backslash\{0\}$, the following inequality holds:

$$
\begin{aligned}
I\left(r Q_{1}\right)= & \frac{1}{2} \int_{-T}^{T}\left|r \dot{Q}_{1}(t)\right|^{2} d t-\int_{-T}^{T} W\left(t, r Q_{1}(t)\right) d t+\int_{-T}^{T}\left(f(t), r Q_{1}(t)\right) d t \\
\leq & \frac{|r|^{2}}{2} \int_{-T}^{T}\left|\dot{Q}_{2}(t)\right|^{2} d t-\left(\frac{\pi^{2}}{2 T^{2}}+\varepsilon_{1}\right)|r|^{2} \int_{-T}^{T}\left|Q_{1}(t)\right|^{2} d t \\
& +|r| \delta m^{1 / 2}+2 T\left(\left(\frac{\pi^{2}}{2 T^{2}}+\varepsilon_{1}\right) \xi^{2}+\zeta\right) \\
= & -\left(\left(\frac{\pi^{2}}{2 T^{2}}+\varepsilon_{1}\right) m-M\right)|r|^{2}+|r| \delta m^{1 / 2}+2 T\left(\left(\frac{\pi^{2}}{2 T^{2}}+\varepsilon_{1}\right) \xi^{2}+\zeta\right),
\end{aligned}
$$

which implies that there exists $r \in R \backslash\{0\}$ such that $\left\|r Q_{1}\right\|>\varrho$ and $I\left(r Q_{1}\right)<0$. Set $e(t)=$ $r Q_{1}(t)$. Then $\|e\|>\varrho$ and $I(e)<0$. 
Lemma 2.3 Suppose that the conditions of Theorem 1.1 hold, then I satisfies the $(C)$ condition.

Proof Assume that $\left\{u_{n}\right\} \subset E$ is a sequence such that $\left\{I\left(u_{n}\right)\right\}$ is bounded and $\left\|I^{\prime}\left(u_{n}\right)\right\|(1+$ $\left.\left\|u_{n}\right\|\right) \rightarrow 0$ as $n \rightarrow \infty$. Then there exists a constant $M_{1}>0$ such that

$$
\left|I\left(u_{n}\right)\right| \leq M_{1}, \quad\left\|I^{\prime}\left(u_{n}\right)\right\|\left(1+\left\|u_{n}\right\|\right) \leq M_{1} .
$$

Now we prove that $\left\{u_{n}\right\}$ is bounded in $E$. Arguing in an indirect way, we assume that $\left\|u_{n}\right\| \rightarrow+\infty$ as $n \rightarrow \infty$. Set $z_{n}=\frac{u_{n}}{\left\|u_{n}\right\|}$, then $\left\|z_{n}\right\|=1$, which implies that there exists a subsequence of $\left\{z_{n}\right\}$, still denoted by $\left\{z_{n}\right\}$, such that $z_{n} \rightarrow z_{0}$ in $E$. By (4) and (8), we get

$$
\begin{aligned}
\left|\int_{R} \frac{W\left(t, u_{n}(t)\right)}{\left\|u_{n}\right\|^{2}} d t-\frac{1}{2}\right| & =\left|-\frac{I\left(u_{n}\right)}{\left\|u_{n}\right\|^{2}}+\int_{R} \frac{\left(f(t), u_{n}(t)\right)}{\left\|u_{n}\right\|^{2}} d t\right| \\
& \leq \frac{M_{1}+\delta\left\|u_{n}\right\|}{\left\|u_{n}\right\|^{2}}
\end{aligned}
$$

which implies that

$$
\left|\int_{R} \frac{W\left(t, u_{n}(t)\right)}{\left\|u_{n}\right\|^{2}} d t\right| \leq 1
$$

for $n$ large enough. The following discussion is divided into two cases.

Case $1: z_{0} \equiv 0$. Let $s>\frac{\delta C_{1}}{2}$. From $(W 1)$ and $(W 4)$, we can deduce that there exists $M_{2}>0$ such that

$$
|W(t, x)| \leq M_{2}\left(|x|^{2}+|x|^{\tau}\right) \quad \forall t \in R
$$

and

$$
|(\nabla W(t, x), x)| \leq M_{2}\left(|x|^{2}+|x|^{\tau}\right) \quad \forall t \in R
$$

By the compactness of the embedding, one can obtain

$$
\limsup _{n \rightarrow \infty} \int_{R}\left|W\left(t, s z_{n}(t)\right)\right| d t \leq M_{2} \limsup _{n \rightarrow \infty} \int_{R}\left(s^{2}\left|z_{n}\right|^{2}+s^{\tau}\left|z_{n}\right|^{\tau}\right) d t=0 .
$$

Set $\lambda_{n}=\frac{s}{\left\|u_{n}\right\|}$. It follows from (8)-(13), (W2), and (W5) that

$$
\begin{aligned}
M_{1} \geq & I\left(u_{n}\right) \\
= & I\left(\lambda_{n} u_{n}\right)+\frac{1-\lambda_{n}^{2}}{2}\left\|u_{n}\right\|^{2} \\
& +\int_{R}\left(W\left(t, \lambda_{n} u_{n}(t)\right)-W\left(t, u_{n}(t)\right)\right) d t+\left(1-\lambda_{n}\right) \int_{R}\left(f(t), u_{n}(t)\right) d t \\
= & I\left(\lambda_{n} u_{n}\right)+\left(\frac{1-\lambda_{n}^{2}}{2}-\lambda_{n}\right)\left\langle I^{\prime}\left(u_{n}\right), u_{n}\right\rangle \\
& +\left(\lambda_{n}\left\|u_{n}\right\|^{2}+\left(\frac{\left(1-\lambda_{n}\right)^{2}}{2}+\lambda_{n}\right) \int_{R}\left(f(t), u_{n}(t)\right) d t\right)
\end{aligned}
$$




$$
\begin{aligned}
& +\int_{R}\left(\left(\frac{1-\lambda_{n}^{2}}{2}-\lambda_{n}\right)\left(\nabla W\left(t, u_{n}(t)\right), u_{n}(t)\right)+W\left(t, \lambda_{n} u_{n}(t)\right)-W\left(t, u_{n}(t)\right)\right) d t \\
\geq & I\left(s z_{n}\right)+o(1)+\left(s-\delta C_{1}\left(\frac{\left(1-\lambda_{n}\right)^{2}}{2}+\lambda_{n}\right)\right)\left\|u_{n}\right\| \\
& +\int_{\left|u_{n}\right| \geq r_{\infty}}\left(\left(\frac{1-\lambda_{n}^{2}}{2}-\lambda_{n}\right)\left(\nabla W\left(t, u_{n}(t)\right), u_{n}(t)\right)\right. \\
& \left.+W\left(t, \lambda_{n} u_{n}(t)\right)-W\left(t, u_{n}(t)\right)\right) d t \\
& +\int_{\left|u_{n}\right| \leq r_{\infty}}\left(\left(\frac{1-\lambda_{n}^{2}}{2}-\lambda_{n}\right)\left(\nabla W\left(t, u_{n}(t)\right), u_{n}(t)\right)\right. \\
& \left.+W\left(t, \lambda_{n} u_{n}(t)\right)-W\left(t, u_{n}(t)\right)\right) d t \\
\geq & \frac{s^{2}}{2}-\int_{R} W\left(t, s z_{n}(t)\right) d t+\int_{R}\left(f(t), s z_{n}(t)\right) d t+o(1) \\
& -d_{2} \int_{\left|u_{n}\right| \geq r_{\infty}} \lambda_{n}^{\mu}\left|u_{n}(t)\right|^{\mu} d t+\int_{\left|u_{n}\right| \leq r_{\infty}}\left(-\frac{\lambda_{n}^{2}+\lambda_{n}}{2}\left(\nabla W\left(t, u_{n}(t)\right), u_{n}(t)\right)\right) d t \\
\geq & \frac{s^{2}}{2}-\delta C_{1} \int_{R}\left|z_{n}(t)\right| d t-d_{2} s^{\mu} \int_{\left|u_{n}\right| \geq r_{\infty}}\left|z_{n}(t)\right|^{\mu} d t+o(1) \\
& +M_{2} \int_{\left|u_{n}\right| \leq r_{\infty}}\left(-\frac{\lambda_{n}^{2}+\lambda_{n}}{2}\left(\left|u_{n}(t)\right|^{2}+\left|u_{n}(t)\right|^{\tau}\right)\right) d t \\
\geq & \frac{s^{2}}{2}-\frac{M_{2}}{2} \int_{\left|u_{n}\right| \leq r_{\infty}}\left(\lambda_{n}^{2}\left|u_{n}(t)\right|^{2}+\lambda_{n}\left|u_{n}(t)\right|^{2}+\lambda_{n}^{2}\left|u_{n}(t)\right|^{\tau}+\lambda_{n}\left|u_{n}(t)\right|^{\tau}\right) d t+o(1) \\
\geq & \frac{s^{2}}{2}-\frac{M_{2}}{2} \int_{\left|u_{n}\right| \leq r_{\infty}}\left(s^{2}\left|z_{n}(t)\right|^{2}+r_{\infty} s\left|z_{n}(t)\right|+r_{\infty}^{\tau-2} s^{2}\left|z_{n}(t)\right|^{2}+r_{\infty}^{\tau-1} s\left|z_{n}(t)\right|\right) d t+o(1) \\
&
\end{aligned}
$$

for $s$ and $n$ large enough, which is a contradiction. Hence $\left\|u_{n}\right\|$ is still bounded in this case, which implies that $\left\{u_{n}\right\}$ is bounded in $E$.

Case 2: $z_{0} \not \equiv 0$. Let $\Omega=\left\{t \in R|| z_{0}(t) \mid>0\right\}$. Then we can see that meas $(\Omega)>0$, where meas denotes the Lebesgue measure. Since $\left\|u_{n}\right\| \rightarrow+\infty$ as $n \rightarrow \infty$ and $\left|u_{n}(t)\right|=\left|z_{n}(t)\right| \cdot\left\|u_{n}\right\|$, then we have $\left|u_{n}(t)\right| \rightarrow+\infty$ as $n \rightarrow \infty$ for a.e. $t \in \Omega$. By (W2), (W3), and Fatou's lemma, we can obtain

$$
\begin{aligned}
\liminf _{n \rightarrow \infty} \int_{R} \frac{W\left(t, u_{n}(t)\right)}{\left\|u_{n}\right\|^{2}} d t & \geq \liminf _{n \rightarrow \infty} \int_{\Omega} \frac{W\left(t, u_{n}(t)\right)}{\left\|u_{n}\right\|^{2}} d t \\
& =\liminf _{n \rightarrow \infty} \int_{\Omega} \frac{W\left(t, u_{n}(t)\right)}{\left|u_{n}\right|^{2}}\left|z_{n}(t)\right|^{2} d t \\
& =+\infty,
\end{aligned}
$$

which contradicts (10). So $\left\|u_{n}\right\|$ is bounded in this case.

By a standard argument, we see that $\left\{u_{n}\right\}$ has a convergent subsequence in $E$. Hence $I$ satisfies the $(C)$ condition. 
Proof of Theorem 1.1 The proof of this theorem is divided into two steps.

Step 1: We show that there exists a function $u_{0} \in E$ such that $I^{\prime}\left(u_{0}\right)=0$ and $I\left(u_{0}\right)<0$. Let $f(t)=\left(f_{1}(t), f_{2}(t), \ldots, f_{N}(t)\right)$, where $f_{i}(t) \in C(R, R)(i=1,2, \ldots, N)$. Since $f \not \equiv 0$, there exists $i_{0} \in[1, N] \cap Z$ such that $f_{i_{0}}(t) \not \equiv 0$. Without loss of generalization, we assume that there exist an interval $(a, b) \subset R$ and a constant $A>0$ such that

$$
f_{i_{0}}(t) \geq A \text { for all } t \in(a, b)
$$

We choose a function $\psi_{0} \in C_{0}^{\infty}(a, b)$ satisfying

$$
\begin{cases}\psi_{0}(t)=-f_{i_{0}}(t), & t \in\left(\frac{3 a+b}{4}, \frac{a+3 b}{4}\right) \\ \psi_{0}(t) \leq 0, & t \in(a, b) \backslash\left(\frac{3 a+b}{4}, \frac{a+3 b}{4}\right), \\ \left|\psi_{0}^{\prime}(t)\right| \leq 2, & t \in(a, b)\end{cases}
$$

Set $\psi(t)=\left(\psi_{1}(t), \psi_{2}(t), \ldots, \psi_{N}(t)\right)$, where $\psi_{j}(t)=0$ for all $j \in[1, N] \cap Z \backslash\left\{i_{0}\right\}$ and $\psi_{i}(t)=$ $\psi_{0}(t)$ for $j=i_{0}$. Therefore, $\psi \in E$ and we can deduce that

$$
\int_{R}(f(t), \psi(t)) d t=\int_{a}^{b} f_{i_{0}}(t) \psi_{0}(t) d t \leq-\int_{\frac{3 a+b}{4}}^{\frac{a+3 b}{4}} f_{i_{0}}^{2}(t) d t \leq-\frac{A^{2}}{2}(b-a)<0 .
$$

Hence we have

$$
\begin{aligned}
I(r \psi) & =\frac{1}{2} \int_{R}|r \dot{\psi}(t)|^{2} d t-\int_{R} W(t, r \psi(t)) d t+\int_{R}(f(t), r \psi(t)) d t \\
& \leq \frac{r^{2}}{2} \int_{R}|\dot{\psi}(t)|^{2} d t-M_{2}\left(r^{2} \int_{R}|\psi(t)|^{2} d t+r^{\tau} \int_{R}|\psi(t)|^{\tau}\right) d t+r \int_{R}(f(t), \psi(t)) d t \\
& <0
\end{aligned}
$$

for $r>0$ small enough. Then we obtain

$$
c_{0}=\inf \left\{I(u): u \in B_{\varrho}\right\}<0 \text {, }
$$

where $\varrho$ is defined in Lemma 2.1 and $B_{\varrho}=\{u \in E \mid\|u\| \leq \varrho\}$. By Ekeland's variational principle, there exists a sequence $\left\{u_{n}\right\} \subset B_{\varrho}$ such that

$$
c_{0} \leq I\left(u_{n}\right) \leq c_{0}+\frac{1}{n}
$$

and

$$
I(w) \leq I\left(u_{n}\right)-\frac{1}{n}\left\|w-u_{n}\right\|
$$

for all $w \in B_{\varrho}$. Then, by a standard procedure, we can show that $\left\{u_{n}\right\}$ is a $(C)$ sequence of $I$. Therefore, it follows from Lemma 2.3 that there exists a function $u_{0} \in E$ such that $I^{\prime}\left(u_{0}\right)=0$ and $I\left(u_{0}\right)<0$.

Step 2: By the mountain pass theorem and Lemmas $2.1-2.3$, there exists $\tilde{u}_{0} \in E$ such that $I^{\prime}\left(\tilde{u}_{0}\right)=0$ and $I\left(\tilde{u}_{0}\right)>0$.

Then we finish the proof of Theorem 1.1. 


\section{Acknowledgements}

The authors would like to thank the editor and the referees for their valuable comments and suggestions, which improved the quality of our paper.

\section{Funding}

The first author is supported by the Scientific Research Foundation of Huaiyin Institute of Technology (15HGZ014

17HGZ004). The second author is supported by the National Natural Science Foundation of China (No. 11626198) and the Youth Science and Technology Innovation Team of Southwest Petroleum University for Nonlinear Systems

(No. 2017CXTD02) and the Science and Technology Innovation Team of Education Department of Sichuan for Dynamical System and its Applications (No. 18TD0013).

\section{Competing interests}

The authors declare that they have no competing interests.

\section{Authors' contributions}

All authors contributed equally to the writing of this paper. All authors read and approved the final manuscript.

\section{Author details}

${ }^{1}$ Faculty of Mathematics and Physics, Huaiyin Institute of Technology, Huai'an, China. ${ }^{2}$ College of Science, Southwest Petroleum University, Chengdu, China. ${ }^{3}$ Institute of Nonlinear Dynamics, Southwest Petroleum University, Chengdu, China.

\section{Publisher's Note}

Springer Nature remains neutral with regard to jurisdictional claims in published maps and institutional affiliations.

Received: 21 February 2018 Accepted: 12 April 2018 Published online: 17 April 2018

\section{References}

1. Ambrosetti, A., Rabinowitz, P.H.: Dual variational methods in critical point theory and applications. J. Funct. Anal. 14 349-381 (1973)

2. Ambrosetti, A., Coti Zelati, V.: Multiple homoclinic orbits for a class of conservative systems. Rend. Semin. Mat. Univ. Padova 89, 177-194 (1993)

3. Batkam, C.J.: Homoclinic orbits of first-order superquadratic Hamiltonian systems. Discrete Contin. Dyn. Syst. 34(9), 3353-3369 (2014)

4. Caldiroli, P., Jeanjean, L.: Homoclinics and heteroclinics for a class of conservative singular Hamiltonian systems. J. Differ. Equ. 136(1), 76-114 (1997)

5. Chen, H.W., He, Z.M.: Infinitely many homoclinic solutions for a class of second-order Hamiltonian systems. Adv. Differ. Equ. 2014, 161 (2014)

6. Ding, Y.H.: Existence and multiplicity results for homoclinic solutions to a class of Hamiltonian systems. Nonlinear Anal. 25(11), 1095-1113 (1995)

7. Ding, Y.H., Li, S.J.: Homoclinic orbits for first order Hamiltonian systems. J. Math. Anal. Appl. 189(2), 585-601 (1995)

8. Ding, Y.H., Lee, C.: Homoclinics for asymptotically quadratic and superquadratic Hamiltonian systems. Nonlinear Anal. (5) 71, 1395-1413 (2009)

9. Daouas, A.: Homoclinic orbits for superquadratic Hamiltonian systems without a periodicity assumption. Nonlinear Anal. 74(11), 3407-3418 (2011)

10. Felmer, P.L., Silva, E.A. de B.: Homoclinic and periodic orbits for Hamiltonian systems. Ann. Sc. Norm. Super. Pisa, Cl. Sci. (4) 26(2), 285-301 (1998)

11. Izydorek, M., Janczewska, J.: Homoclinic solutions for a class of the second order Hamiltonian systems. J. Differ. Equ. 219(2), 375-389 (2005)

12. Izydorek, M., Janczewska, J.: Homoclinic solutions for nonautonomous second-order Hamiltonian systems with a coercive potential. J. Math. Anal. Appl. 335(2), 1119-1127 (2007)

13. Korman, P. Lazer, A.C.: Homoclinic orbits for a class of symmetric Hamiltonian systems. Electron. J. Differ. Equ. 1994, 1 (1994)

14. LV, Y., Tang, C.-L.: Existence and multiplicity of homoclinic orbits for second-order Hamiltonian systems with superquadratic potential. Abstr. Appl. Anal. 2013, Article ID 328630 (2013)

15. Lv, X., Lu, S., Yan, P.: Existence of homoclinic solutions for a class of second-order Hamiltonian systems. Nonlinear Anal. 72(1), 390-398 (2010)

16. Mawhin, J., Willem, M.: Critical Point Theory and Hamiltonian Systems. Applied Mathematical Sciences, vol. 74. Springer, New York (1989)

17. Ou, Z.-Q., Tang, C.-L.: Existence of homoclinic solution for the second order Hamiltonian systems. J. Math. Anal. Appl. 291(1), 203-213 (2004)

18. Paturel, E.: Multiple homoclinic orbits for a class of Hamiltonian systems. Calc. Var. Partial Differ. Equ. 12(2), 117-143 (2001)

19. Rabinowitz, P.H.: Homoclinic orbits for a class of Hamiltonian systems. Proc. R. Soc. Edinb., Sect. A 114(1-2), 33-38 (1990)

20. Rabinowitz, P.H., Tanaka, K.: Some results on connecting orbits for a class of Hamiltonian systems. Math. Z. 206(3), 473-499 (1991)

21. Rabinowitz, P.H.: Minimax Methods in Critical Point Theory with Applications to Differential Equations. CBMS, Regional Conf. Ser. in Math., vol. 65. Amer. Math. Soc., Providence (1986)

22. Salvatore, A.: Homoclinic orbits for a special class of nonautonomous Hamiltonian systems. In: Proceedings of the Second World Congress of Nonlinear Analysts, Part 8, Athens, 1996. Nonlinear Anal., vol. 30, pp. 4849-4857 (1997) 
23. Sun, J., Chen, H., Nieto, J.J.: Homoclinic orbits for a class of first-order nonperiodic asymptotically quadratic Hamiltonian systems with spectrum point zero. J. Math. Anal. Appl. 378, 117-127 (2011)

24. Sun, J., Chen, H., Nieto, J.J.: Homoclinic solutions for a class of subquadratic second-order Hamiltonian systems. J. Math. Anal. Appl. 373, 20-29 (2011)

25. Sun, J., Wu, T.F.: Multiplicity and concentration of homoclinic solutions for some second order Hamiltonian systems. Nonlinear Anal. 114, 105-115 (2015)

26. Tang, X.H., Xiao, L.: Homoclinic solutions for a class of second order Hamiltonian systems. Nonlinear Anal. 71, 1140-1152 (2009)

27. Tang, X.H., Xiao, L.: Homoclinic solutions for nonautonomous second-order Hamiltonian systems with a coercive potential. J. Math. Anal. Appl. 351(2), 586-594 (2009)

28. Tang, C.-L., Wu, X.-P.: Periodic solutions for a class of new superquadratic second order Hamiltonian systems. Appl. Math. Lett. 34, 65-71 (2014)

29. Tao, Z.-L., Tang, C.-L.: Periodic and subharmonic solutions of second-order Hamiltonian systems. J. Math. Anal. Appl. 293(2), 435-445 (2004)

30. Tanaka, K.: Homoclinic orbits in a first order superquadratic Hamiltonian system: convergence of subharmonic orbits. J. Differ. Equ. 94(2), 315-339 (1991)

31. Wan, L.-L., Tang, C.-L.: Existence and multiplicity of homoclinic orbits for second order Hamiltonian systems without (AR) condition. Discrete Contin. Dyn. Syst. 15, 255-271 (2011)

32. Wu, D.-L., Li, C., Yuan, P.F.: Multiplicity solutions for a class of fractional Hamiltonian systems with concave-convex potentials. Mediterr. J. Math. 15, 35 (2018)

33. Wu, D.-L., Tang, C.-L., Wu, X.-P.: Homoclinic orbits for a class of second-order Hamiltonian systems with concave-convex nonlinearities. Electron. J. Qual. Theory Differ. Equ. 6, 6 (2018)

34. Yang, M.H., Han, Z.Q.: The existence of homoclinic solutions for second-order Hamiltonian systems with periodic potentials. Nonlinear Anal., Real World Appl. 12(5), 2742-2751 (2011)

35. Ye, Y.-W., Tang, C.-L.: Periodic and subharmonic solutions for a class of superquadratic second order Hamiltonian systems. Nonlinear Anal. 71(5-6), 1-15 (2009)

36. Xu, J.F., O'Regan, D., Zhang, K.Y.: Multiple solutions for a class of fractional Hamiltonian systems. Fract. Calc. Appl. Anal. $18,48-63(2015)$

37. Zhang, X., Tang, X.: Subharmonic solutions for a class of non-quadratic second order Hamiltonian systems. Nonlinear Anal., Real World Appl. 13(1), 113-130 (2012)

38. Zhang, X., Tang, X.: Existence of subharmonic solutions for non-quadratic second-order Hamiltonian systems. Bound. Value Probl. 2013, 139 (2013)

39. Zhang, Z., Yuan, R.: Homoclinic solutions for some second order non-autonomous Hamiltonian systems without the globally superquadratic condition. Nonlinear Anal. 72(3), 1809-1819 (2010)

40. Zhang, Z., Yuan, R.: Two almost homoclinic solutions for a class of perturbed Hamiltonian systems without coercive conditions. Math. Model. Anal. 20(1), 112-123 (2015)

41. Zhang, X.: Homoclinic orbits for a class of p-Laplacian systems with periodic assumption. Electron. J. Qual. Theory Differ. Equ. 2013, 67 (2013)

\section{Submit your manuscript to a SpringerOpen ${ }^{\circ}$ journal and benefit from:}

- Convenient online submission

- Rigorous peer review

- Open access: articles freely available online

- High visibility within the field

- Retaining the copyright to your article

Submit your next manuscript at $\gg$ springeropen.com 